\title{
Optimal Voltage Control Algorithm of Small Hydropower Generators for Voltage Stabilization in Distribution System with Large Scale PV Systems
}

\author{
Hongyeol Choi ${ }^{1}$, Hudong Lee ${ }^{2}$, Marito Ferreira ${ }^{3}$, Jihyun Park ${ }^{4}$, and Daeseok Rho ${ }^{5}$ \\ Korea University of Technology and Education, Korea \\ ${ }^{1}$ hong@kwater.or.kr, ${ }^{2}$ lhd714@koreatech.ac.kr, ${ }^{3}$ ferr12345@koreatech.ac.kr, \\ 4dsois94@koreatech.ac.kr, ${ }^{5}$ dsrho@koreatech.ac.kr
}

\begin{abstract}
According to the government's policy to demonstrate and expand the renewable energy sources, distributed generators such as $P V$ (photovoltaic) and WP (wind power) systems are installed and operated in distribution systems. However, there are many issues related to power quality problems including over-voltage and under-voltage of customers. Especially Blackouts - the total loss of electrical power - serve as a reminder of how dependent the modern world and particularly urban areas have become on electricity and the appliances it powers. In order to overcome these problems, the electric power company have installed a Step Voltage Regulator $(S V R)$ in primary feeders interconnected with distributed generators, and also have established the technical guidelines for the distributed generators to stabilize the customer voltages in distribution systems. However, it is difficult to maintain the customer voltages within allowable limit. Therefore, this paper analyzes the problem of voltage control by SVR in distribution system with large scale PV systems, and presents operating range and voltage control limit of Small Hydropower Generator (SHG) with PF control function. In addition, this paper evaluates the voltage impact in primary feeders depending on voltage control modes of SHG, and then proposes optimal voltage control algorithm. Furthermore, from the simulation results base on the proposed algorithm, it is confirmed that it can contribute to voltage stabilization in distribution system interconnected with large scale PV systems.
\end{abstract}

Keywords: Small hydropower generator, Optimal voltage control, Voltage stabilization, Reactive power

\section{Introduction}

Recently, the government is pushing a green growth policy as one of the next generation industry and actively performing the demonstration project of $11 \%$ renewable sources among total energy by 2030. According to the activity plan of renewable energy sources, local governments and renewable energy producers are actively installing and operating large scale PV systems in the distribution system. However, when the PV systems were interconnected and operated in distribution system, many problems related to power quality such as voltage fluctuation in distribution system are being occurred [1][2][3]. Especially Blackouts-the total loss of electrical power-serve as a reminder of how dependent the modern world and

Article history:

Received (June 4, 2019), Review Result (August 13, 2019), Accepted (September 15, 2019) 
particularly urban areas have become on electricity and the appliances it powers [4]. In order to solve this problem, the power company have installed SVR in order to maintain customer voltages within allowable voltage limits in distribution system interconnected with PV systems [5][6][7]. In addition, distributed generations, renewable energy sources, should be kept at more than $90 \%$ of PF in accordance with the grid interconnection codes, and in case of technically necessary, the upper and lower limits of PF should be controlled according to technical guideline of power company, and also PF of the distributed generations have to not become leading PF at point of distribution system which is lagging PF at distributed generations[8]. However, despite of such voltage regulators and technical guidelines, there are many problems associated with the voltage fluctuation and power quality when large scale PV systems and SHGs are connected with distribution system. Therefore, this paper analyzes the problem of voltage control by SVR in distribution system with large scale PV systems, and presents operating range and voltage control limit of SHG with PF control function. And also, this paper evaluates the voltage impact in primary feeders depending on voltage control modes of SHG, and then proposes optimal voltage control algorithm. Furthermore, from the simulation results base on the proposed algorithm, it is confirmed that it is practical and effective to voltage stabilization in distribution system interconnected with large scale PV systems.

\section{Optimal voltage control algorithm of SHG for voltage stabilization}

AVR mode among several voltage control modes is proper control mode to mitigate voltage variation at PCC. However, this control mode also is not enough to properly mitigate voltage variation at PCC. If system voltage at PCC (VL) is higher than the setting voltage (Vs), AVR mode is firstly to adjust voltage of SHG until the setting voltage after starting of generator, and to synchronize SHG at PCC with being compared to voltage at PCC (VL) and then voltage of SHG (VG) is constantly operated with variation of system voltage at PCC as shown in [Figure 1]. Therefore, SHG may cause overvoltage at PCC according to its reverse power flow.

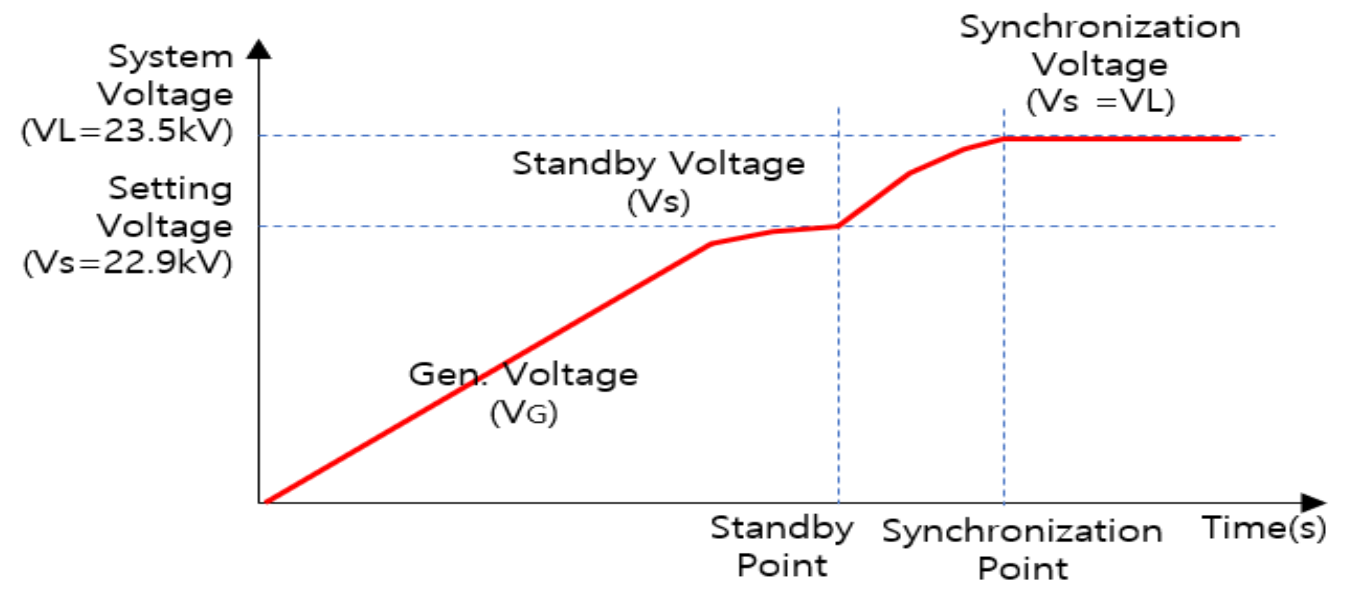

Figure 1. Concept of existing voltage control method

Existing algorithm of voltage control is supposed to regulate voltage of SHG (VSET) following system voltage at PCC. The algorithm is not enough to properly mitigate voltage variation at PCC because voltage of SHG is normally determined according to voltage conditions at PCC with reverse power of PV systems and load variation. While voltage of SHG is synchronized and followed by variation of voltage at PCC, proposed algorithm is scheduled 
to properly regulate the voltage of SHG (VSET) at set point regardless of voltage variation at PCC by replacing the setting value of exciter with setting voltage in order to stabilize system voltage at PCC. Therefore, the proposed algorithm of optimal voltage control are as follows.

[Step 1] Build up the voltage of SHG (VG) with initial set value (Vpre) of exciter from starting point to standby point in [Figure 1].

[Step 2] Control voltage of SHG by synchronizer from standby point to synchronization point, which is constantly followed by system voltage of PCC (VL) regardless of system voltage variation.

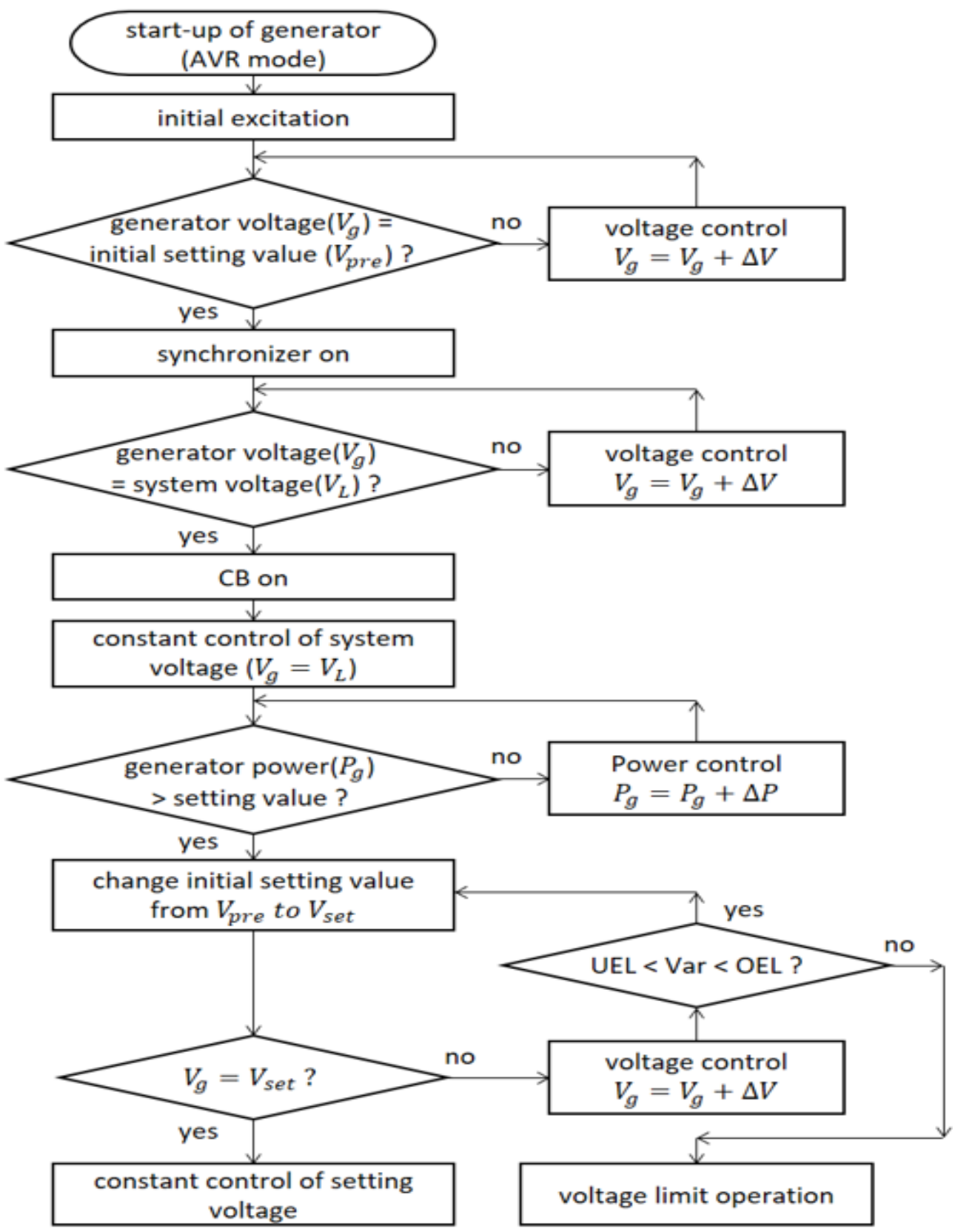

Figure 2. Optimal voltage control algorithm of SHG 
[Step 3] Replace setting value of excitation controller with setting voltage (Vset) if output of SHG is more than scheduled value from synchronization point to set point and then regulate the voltage of SHG to setting voltage with exciter control.

[Step 4] Regulate voltage of SHG within the capability curve of output power, which is operated between OEL (overexcitation limit) and UEL (under excitation limit).

[Step 5] Even if SHG violates capability curve of output power, the voltage of SHG is maintained with limited conditions.

\section{Case studies}

\subsection{Verification of optimal algorithm by excitation system simulator}

The proposed optimal algorithm of voltage control is implemented at existing simulator of the excitation system called RTS-7000 [17], and its performance characteristics are demonstrated in [Figure 3]. [Figure 3] shows the case where system voltage at PCC is higher than setting voltage of SHG, which is characteristics of field voltage, field current, reactive power and voltage variation at SHG. Where, (1) of [Figure 3] shows synchronization point and (2) is the optimal voltage control point. And also "Section I" of Figure 3 is a section which the rated voltage of SHG is determined by field voltage and current after start-up and "Section II" is a section in which voltage of SHG is increased by supplying reactive power to follow system voltage at PCC after synchronization of SHG. Furthermore, "Section III" is a section which the rated voltage of SHG is maintained by absorbing reactive power with optimal voltage control if system voltage in the PCC is higher than one of SHG. Therefore, it is confirmed that the proposed algorithm is useful for voltage control in the distribution system.

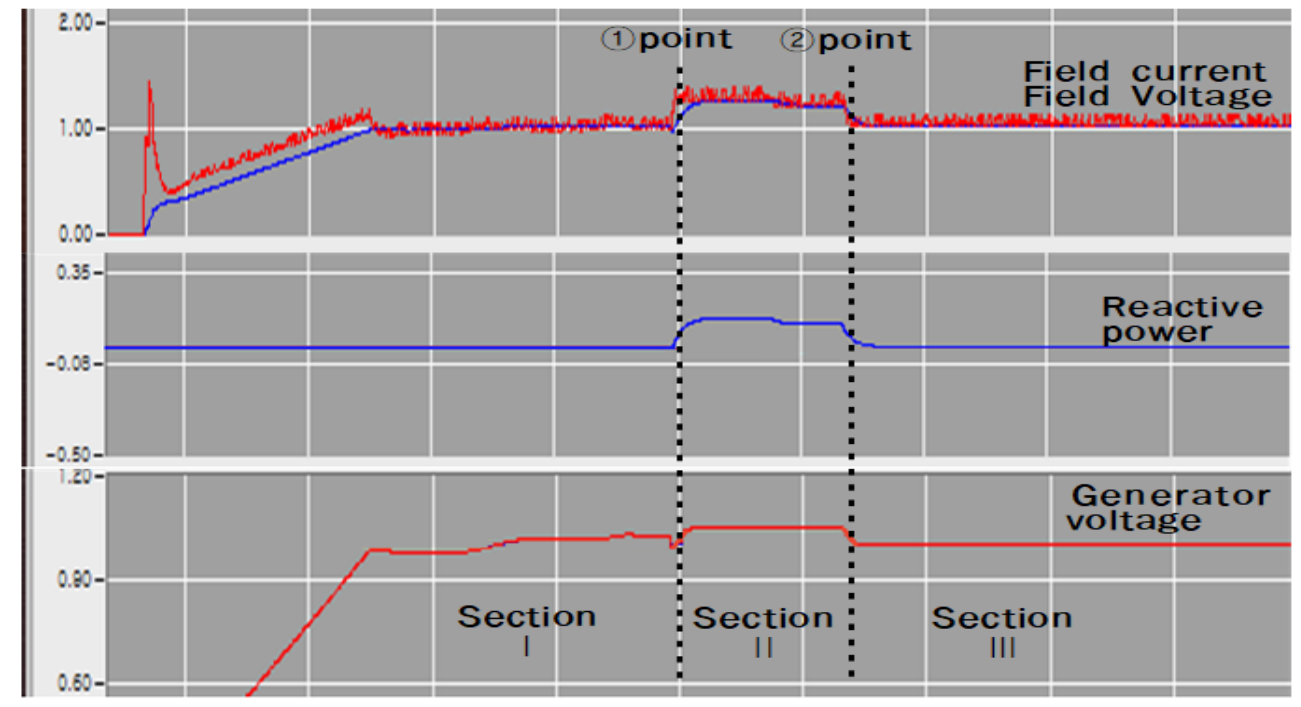

Figure 3. Voltage profile in case of higher system voltage than setting voltage of SHG

[Figure 4] shows the case where system voltage at PCC is lower than setting voltage of SHG. Field current and active power is controlled based on the optimal algorithm, and then system voltage at PCC can be maintained within allowable limit in section III as shown in [Figure 4]. Therefore, it is confirmed that the proposed algorithm is useful for voltage control in distribution system. 


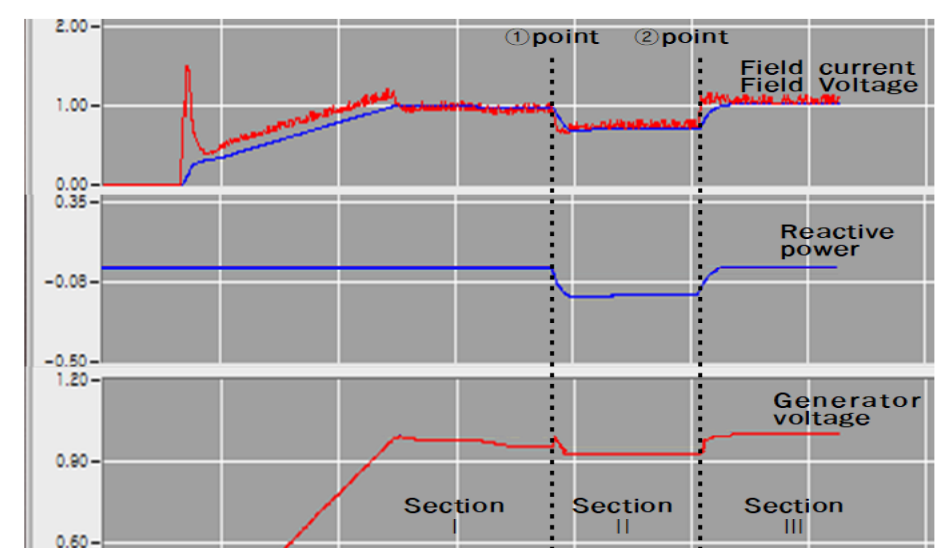

Figure 4. Voltage profile in case of lower system voltage than setting voltage of SHG

\subsection{Control characteristics of optimal algorithm in real system}

\subsubsection{Simulation condition}

[Figure 5] shows configuration of $\mathrm{Y} \mathrm{D} / \mathrm{L}$ (distribution line) in $\mathrm{H} \mathrm{S/S} \mathrm{(substation)} \mathrm{which} \mathrm{is}$ interconnected with large scale PV systems and SHG, as shown in [Table 1]. Specifically, total length of $\mathrm{D} / \mathrm{L}$ is as long as $40.5 \mathrm{~km}$, and $1.8 \mathrm{MW}$ of $\mathrm{SHG}$ is located at $13.2 \mathrm{~km}$ from $\mathrm{S} / \mathrm{S}$. And also, total of 6.3MW of PV systems are installed through the D/L. Furthermore, a range of daily load profile is from 3.7MW at off-peak load to 9.8MW at peak load. In addition, the voltage of primary feeder can be significantly maintained higher than nominal voltage when large scale PV systems is operated.

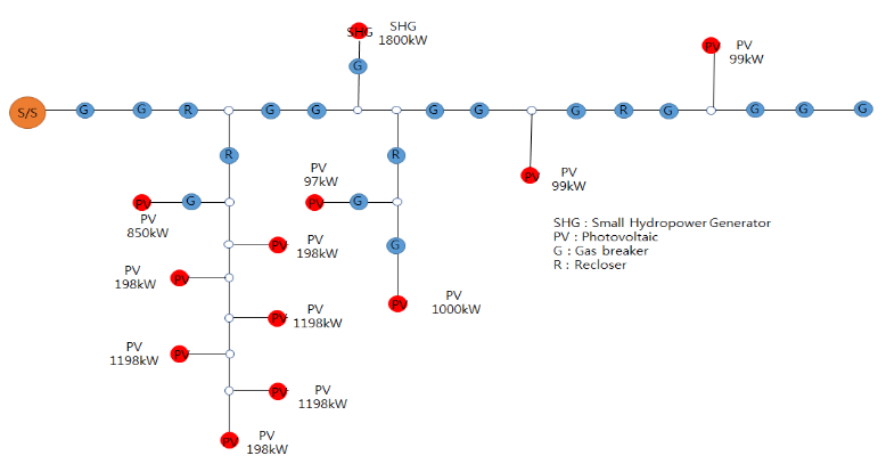

Figure 5. Real distribution system with PV systems

\subsubsection{Control characteristics of optimal algorithm}

Based on the proposed optimal voltage control algorithm of SHG, voltage characteristics at PCC is demonstrated in [Figure 6]. The [Figure 6] (a) shows characteristics of reactive power variation and [Figure 6] (b) is characteristics of voltage variation at SHG. On condition that system voltage at PCC is $23.2 \mathrm{kV}$. Reactive power of generator is controlled from $17 \mathrm{kV}$ ar to $60 \mathrm{kVar}$ by resetting generator voltage to $23 \mathrm{kV}$ through the proposed optimal control algorithm of SHG. It is found that system voltage at PCC is decreased to $23 \mathrm{kV}$. Therefore, it is confirmed that proposed optimum voltage control algorithm can constantly control voltage at PCC of SHG, 
and it can contribute to voltage stabilization of distribution system even though large scale PV system is operated.

Table 1. Parameters of distribution line

\begin{tabular}{|c|c|}
\hline Items & Contents \\
\hline Main feeder length & $40.5 \mathrm{~km}$ \\
\hline Location of SHG & $13.2 \mathrm{~km}$ \\
\hline Total capacity of PV systems & $6.333 \mathrm{MW}$ \\
\hline Capacity of SHG & $1.8 \mathrm{MW}$ \\
\hline Peak load & $9.8 \mathrm{MW}$ \\
\hline Off-peak load & $3.7 \mathrm{MW}$ \\
\hline
\end{tabular}

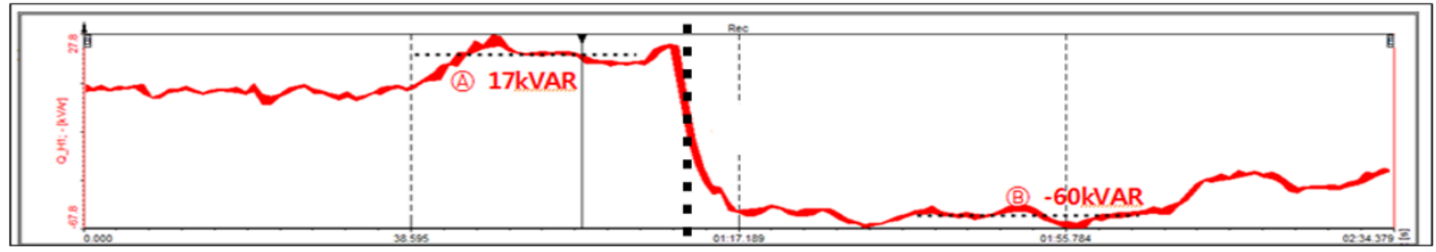

(a) Reactive power

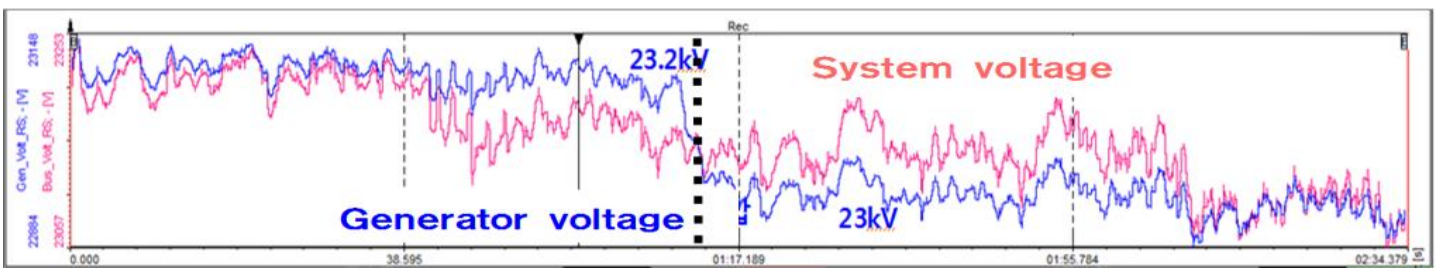

(b) Generator and system voltage

Figure 6. Characteristics of reactive power and system voltage

\section{Conclusions}

This paper proposes an optimal voltage control algorithm of SHG to overcome problems of voltage control in a distribution system interconnected with large scale PV systems. And also, this paper implements excitation system simulator based on the proposed optimal control algorithm. The main results of this paper are summarized as follows.

(1) This paper has implemented excitation system simulator using RTS-7000 based on the proposed optimal control algorithm and has performed its characteristics. If system voltage at PCC is higher than setting voltage of SHG, system voltage is constantly maintained to setting voltage by controlling field current and reactive power with proposed optimal voltage control algorithm. Therefore, it is confirmed that the proposed algorithm is useful for voltage control in distribution system.

(2) It is clear that the proposed optimal voltage algorithm can control reactive power of SHG to regulate the system voltages of PCC which are normally in overvoltage state, if large-scaled of PV system is interconnected in distribution system.

(3) It is confirmed that optimum voltage control algorithm proposed in this paper can constantly control voltage at PCC even if large scale PV system is connected with distribution system, and the algorithm can contribute voltage stabilization of distribution system. 


\section{Acknowledgements}

This work was supported by the power generation \& electricity delivery core technology program of the Korea institute of energy technology evaluation and planning (KETEP) granted financial resource from the ministry of trade, industry \& energy, republic of Korea (No.20172410100030 and No.20182410105070).

\section{References}

[1] The Korea Institute of Electrical Engineers, "New distribution engineering," Bookshill, (2014)

[2] Roger C. Dugan, Mark F. McGranaghan, Surya Santoso, and H. Wayne Beaty, "Electrical power system quality," second edition, (2004)

[3] IEEE 1547.6, IEEE Standard conformance test procedures for equipment interconnecting distributed resources with electric power system, June, (2009)

[4] Hugh Byrd and Steve Matthewman, "Exergy and the city: The technology and sociology of power (failure)," Journal of Urban Technology, vol.21, no.3, pp.85-102, (2014) DOI: 10.1080/10630732.2014.940706

[5] Byungki Kim and Daeseok Rho, "Optimal operation methods of protection devices in distribution systems with PV systems,” Smart Computing Review, vol.4, no.6, pp.506-514, (2014) DOI: 10.5370/KIEE.2011.60.8.1485

[6] Byungki Kim., "A study on the voltage regulation method of distribution system using step voltage regulator and energy storage system," Doctor thesis, (2015)

[7] YangHyun Nam, "Study on the large-scale adoption method of distribution system interconnected with PV system by energy storage system," vol.67, no.8, pp.1031-1039, (2018) DOI: 10.5370/KIEE.2018.67.8.1031

[8] Technical guidelines for the distributed generators, KEPCO, (2017) 
Optimal Voltage Control Algorithm of Small Hydropower Generators for Voltage Stabilization in Distribution System with Large Scale PV Systems

This page is empty by intention. 